TITLE:

\title{
A Solvable Chaotic Map ofq-Phase Transitions Caused by Diverging Local Expansion Rates
}

\author{
$\operatorname{AUTHOR}(\mathrm{S})$ :
}

Miyazaki, Syuji

\section{CITATION:}

Miyazaki, Syuji. A Solvable Chaotic Map ofq-Phase Transitions Caused by Diverging Local Expansion Rates. Journal of the Physical Society of Japan 2013, 82(12): 125002.

ISSUE DATE:

2013-12-15

URL:

http://hdl.handle.net/2433/254368

\section{RIGHT:}

(C)2013 The Physical Society of Japan; This is not the published version. Please cite only the published version.; この論文は出版社版でありませ ん。引用の際には出版社版をご確認ご利用ください。 


\title{
A Solvable Chaotic Map of $q$-Phase Transitions Caused by Diverging Local Expansion Rates
}

\author{
Syuji MrYazaKi* \\ Graduate School of Informatics, Kyoto University, Yoshida-Honmachi, Sakyo-ku, Kyoto 606-8501, Japan
}

\begin{abstract}
KEYWORDS: deterministic chaos, large deviation, local expansion rate, rate function, topological conjugacy, thermodynamic formalism, crisis
\end{abstract}

In chaotic dynamical systems, non-hyperbolicity or bifurcations such as an onset of type-I intermittency (a tangent bifurcation) and a crisis, which can be observed, for example, at both ends of the period-three window of the logistic map, cause characteristic large fluctuations in a finite range of the local expansion rate, whose long time average is equal to the maximum Lyapunov exponent. This appears as a non-analytic rate function of the local expansion rate. Some researchers call it a " $q$-phase transition".?)

For a one-dimensional chaotic map $x_{n+1}=f\left(x_{n}\right)$, the local expansion rate $\lambda\left(x_{n}\right)$ is given by $\lambda\left(x_{n}\right)=\log \left|\frac{d f\left(x_{n}\right)}{d x_{n}}\right|$. If the map has a smooth extremum at $x=x_{*}, \lambda\left(x_{*}\right)$ goes to negative infinity. Although a smooth extremum is a special case of non-hyperbolicity in a one-dimensional map, diverging local expansion rates due to non-hyperbolicity in general chaotic dynamical systems such as higher-dimensional maps and differential equations also cause a non-analytic rate function of the local expansion rate?

The Lorenz plot with the classical parameters?) shaped maximum, at which the local expansion rate goes to positive infinity. Szépfalusy et al. studied thermodynamics (large deviations) of the local expansion rate in such Lorenztype maps to derive some phase transitions. ?)

In this short paper, we consider a hyperbola-shaped onedimensional map, which is an iteration of the Newton method of solving $x^{2}+1=0$, and is topologically conjugate to the Bernoulli shift. Its invariant density turns out to be a Cauchy distribution. By use of this map, we obtain an exact rate function of the local expansion rate, which has a non-analytic point. Owing to the limited space of Short Notes, readers are kindly requested to refer to the literature ${ }^{\text {?) }}$ for a detailed introduction and detailed derivation of thermodynamic functions.

Applying the Newton method to the equation $x^{2}+1=0$, which has no real roots, we have the iterative map

$$
x_{t+1}=\frac{1}{2}\left(x_{t}-\frac{1}{x_{t}}\right) \quad(-\infty<x<\infty),
$$

which is topologically conjugate to the Bernoulli shift $z_{t+1}=$ $2 z_{t}(\bmod 1)(0 \leq z<1)$, whose invariant density is uniform, via the variable transformation $x=-\cot \pi z$, as indicated by Devaney.? This map is not contained in the list of solvable chaotic maps in the literature.?)

The invariant density and the Lyapunov exponent are not mentioned by Devaney,? which can be derived straightfor- wardly. Owing to the conservation of probability $p(x) d x=$ $1 \cdot d z$, the invariant density is given by the Cauchy distribution $p(x)=\frac{1}{\pi\left(1+x^{2}\right)}$

By use of the transform $x(z)=-\cot \pi z$ and the conservation of probability $p(x) d x=1 \cdot d z$, the Lyapunov exponent $\Lambda^{\infty}$ can be obtained from the average of the local expansion rate with respect to the invariant density as

$$
\begin{aligned}
& \Lambda^{\infty}=\log 2=\int_{-\infty}^{\infty}\left(\log \frac{1+x^{2}}{x^{2}}-\log 2\right) p(x) d x \\
& =\int_{0}^{1}\left(\log \frac{1+\cot ^{2} \pi z}{\cot ^{2} \pi z}-\log 2\right) d z=-2 I-\log 2,
\end{aligned}
$$

where the following relations are used:

$$
\begin{aligned}
I & =\frac{2}{\pi} \int_{0}^{\pi / 2} \log \left(\cos \theta^{\prime}\right) d \theta^{\prime} \\
& =\frac{2}{\pi} \int_{\pi / 2}^{0} \log \left(\cos \left(\frac{\pi}{2}-\alpha\right)\right) d\left(\frac{\pi}{2}-\alpha\right) \\
& =\frac{2}{\pi} \int_{0}^{\pi / 2} \log (\sin \alpha) d \alpha \\
2 I & =\frac{2}{\pi} \int_{0}^{\pi / 2} \log \left(\cos \theta^{\prime}\right) d \theta^{\prime}+\frac{2}{\pi} \int_{0}^{\pi / 2} \log \left(\sin \theta^{\prime}\right) d \theta^{\prime} \\
& =\frac{2}{\pi} \int_{0}^{\pi / 2} \log \left(\frac{\sin 2 \theta^{\prime}}{2}\right) d \theta^{\prime}=I-\log 2,
\end{aligned}
$$

leading to $I=-\log 2$, where the relation $\theta^{\prime}=\frac{\pi}{2}-\alpha$ is used.

Several frameworks of the thermodynamic formalism of the temporal fluctuation have been introduced. We hereafter adopt the formalism introduced by Mori et al. ${ }^{\text {) }}$ The average of the local expansion rates over $n$ steps $\Lambda_{n}\left(x_{0}\right)=\frac{1}{n} \sum_{k=0}^{n-1} \lambda\left(x_{k}\right)$ also fluctuates. When we introduce the probability $P(\Lambda ; n) d \Lambda$ for $\Lambda_{n}\left(x_{0}\right)$ to take a value in the interval $[\Lambda, \Lambda+d \Lambda]$ for a given $n$, the probability density function is given by $P(\Lambda ; n)=$ $\left\langle\delta\left(\Lambda_{n}(x)-\Lambda\right)\right\rangle$, where $\delta(g)$ is the $\delta$-function of $g$ and $\langle f(x)\rangle$ is the average over the natural invariant measure $\int f(x) p(x) d x$. The rate function of the local expansion rate $\psi(\Lambda)$ is defined by the limit

$$
\psi(\Lambda)=-\lim _{n \rightarrow \infty} \frac{1}{n} \log \frac{P(\Lambda ; n)}{P\left(\Lambda^{\infty} ; n\right)} .
$$

For a real parameter $q(-\infty<q<\infty)$ conjugate to $\Lambda$, we in- 
troduce the partition function $Z_{n}(q)=\left\langle\exp \left(-n(q-1) \Lambda_{n}(x)\right)\right\rangle$, the free-energy-like function $\Phi(q)=-\lim _{n \rightarrow \infty} \frac{1}{n} \log Z_{n}(q)$, and the magnetization-like function $\Lambda(q)=\frac{d \Phi(q)}{d q}$. Among these functions, the following Legendre-transform-like relations hold: $\Phi(q)=\psi(\Lambda(q))+(q-1) \Lambda(q)$ and $\psi(\Lambda)=\Phi(q(\Lambda))-$ $(q(\Lambda)-1) \Lambda$, where $q(\Lambda)$ is the inverse function of $\Lambda(q)$.

The finite-time average of the local expansion rates over $n$ steps is obtained from the chain rule as

$$
\left|\frac{d x_{n}}{d x_{0}}\right|=\left|\frac{d x_{n}}{d x_{n-1}} \frac{d x_{n-1}}{d x_{n-2}} \cdots \frac{d x_{1}}{d x_{0}}\right|=2^{n}\left|\frac{\sin ^{2} \pi z_{0}}{\sin ^{2} \pi z_{n}}\right|,
$$

where the relation $\frac{d x}{d z}=-\frac{\pi}{\sin ^{2} \pi z}$ is used. Its partition function is given by

$$
\begin{aligned}
Z_{n}(q) & =\int_{0}^{1} h\left(z_{0}\right) d z_{0}=\left(\int_{0}^{1 / 2}+\int_{1 / 2}^{1}\right) h\left(z_{0}\right) d z_{0} \\
& =2 \int_{0}^{1 / 2} h\left(z_{0}\right) d z_{0}=2 \int_{1 / 2}^{1} h\left(z_{0}\right) d z_{0},
\end{aligned}
$$

where the integrand $h\left(z_{0}\right)$ is given by $h\left(z_{0}\right)=$ $2^{n(1-q)}\left|\frac{\sin ^{2} \pi z_{0}}{\sin ^{2} \pi z_{n}}\right|^{1-q}$, and the conservation of probability $(p(x) d x=1 \cdot d z)$ is used.

For $1-q>0$, i.e., $q<1$, zero points of $\sin \pi z_{n}$ make a singular contribution to the integral. If $z_{n}$ is found in the interval $0<z_{n}<\delta$, the initial point $z_{0}$ exists in the interval $1 / 2<z_{0}<1 / 2+2^{-n} \delta$ with a width of $2^{-n} \delta$ (if $z_{n}$ is found in the interval $1-\delta<z_{n}<1, z_{0}$ exists in the interval $1 / 2-2^{-n} \delta<z_{0}<1 / 2$ with a width of $2^{-n} \delta$ ). From the approximation $\sin ^{2} \pi z_{n} \sim \sin ^{2} \pi \delta \sim(\pi \delta)^{2}$, we have

$$
\begin{aligned}
Z_{n}(q) & \sim 2\left(2^{n(1-q)} \delta^{-2(1-q)} \cdot 2^{-n} \delta c_{2}^{\prime}+2^{n(1-q)} c_{0}^{\prime}\right) \\
& =2^{-n q} \delta^{2 q-1} c_{2}+2^{n(1-q)} c_{0},
\end{aligned}
$$

where $c_{2}^{\prime}, c_{0}^{\prime}, c_{2}$, and $c_{0}$ are constants of order $O\left(\delta^{0}\right)$, and the second term originates from a non-singular contribution to the integral. For $q<1$ and $2 q-1<0$, i.e., $q<1 / 2$, we have $Z_{n}(q) \rightarrow \infty$ as $\delta \rightarrow 0$. For $q<1$ and $2 q-1>0$, i.e., $1 / 2<$ $q<1$, we have $Z_{n}(q) \sim 2^{n(1-q)}$ as $\delta \rightarrow 0 \mathrm{D}$

For $1-q<0$, i.e., $q>1$, zero points of $\sin \pi z_{0}$ make a singular contribution to the integral. When we set $z_{j}=\epsilon_{j}$ and $\epsilon_{j}=2^{j-n} \epsilon \ll 1$ for $0<z_{0}<2^{-n} \epsilon$ (when we set $z_{j}=1-\epsilon_{j}$ and $\epsilon_{j}=2^{j-n} \epsilon \ll 1$ for $\left.1-2^{-n} \epsilon<z_{0}<1\right)$, and we use the estimation $\sin ^{2} \pi z_{j}=\sin ^{2} \pi \epsilon_{j} \sim\left(\pi \epsilon_{j}\right)^{2}=2^{-2 n} \epsilon^{2}$, we have

$$
\begin{aligned}
Z_{n}(q) & =2\left(\int_{0}^{2^{-n} \epsilon}+\int_{2^{-n} \epsilon}^{1 / 2}\right) h\left(z_{0}\right) d z_{0} \\
& =2\left(2^{n(1-q)}\left(2^{-2 n} \epsilon^{2}\right)^{1-q} 2^{-n} \epsilon c_{1}^{\prime}+2^{n(1-q)} c_{0}^{\prime}\right) \\
& =2^{n(q-2)} \epsilon^{3-2 q} c_{1}+2^{n(1-q)} c_{0},
\end{aligned}
$$

where $c_{1}^{\prime}, c_{0}^{\prime}, c_{1}$, and $c_{0}$ are constants of order $O\left(\epsilon^{0}\right)$, and the second term originates from a non-singular contribution to the integral. The sign of the power of $\epsilon$ determines which is the main contribution to the integral between the first and second terms for $\epsilon \rightarrow 0$. We have $Z_{n}(q) \sim 2^{n(1-q)}$ for $q>1$ and $3-2 q>0$, i.e., $1<q<3 / 2$, and $Z_{n}(q) \sim 2^{n(q-2)}$ for $q>1$ and $3-2 q<0$, i.e., $q>3 / 2$.

In short, the partition function is obtained as $Z_{n}(q) \sim \infty$ for $q<1 / 2, \exp [n(1-q) \log 2]$ for $1 / 2<q<3 / 2$, and $\exp [n(q-$ 2) $\log 2$ ] for $q>3 / 2$, so that the free-energy-like function and the magnetization-like function are given by $\Phi(q)=-\infty$ for $q<1 / 2,(q-1) \log 2$ for $1 / 2<q<3 / 2$, and $-(q-2) \log 2$ for $q>3 / 2 ; \Lambda(q)=\infty$ for $q<1 / 2, \log 2$ for $1 / 2<q<3 / 2$, and $-\log 2$ for $q>3 / 2$. The inverse function of the latter is given by $q(\Lambda)=1 / 2$ for $\Lambda>\log 2,3 / 2$ for $-\log 2<\Lambda<\log 2$, and $\infty$ for $\Lambda<-\log 2$. The Legendre-transform-like relation $\psi(\Lambda)=\Phi(q(\Lambda))-(q(\Lambda)-1) \Lambda$ yields the rate function of the local expansion rate $\psi(\Lambda)$ as follows:

$$
\begin{aligned}
\psi(\Lambda)= & \frac{\Lambda}{2}-\frac{\log 2}{2} \quad\left(\Lambda>\Lambda^{\infty}=\log 2\right) \\
& \frac{\log 2}{2}-\frac{\Lambda}{2} \quad\left(-\log 2<\Lambda<\Lambda^{\infty}=\log 2\right) \\
& \infty \quad(\Lambda<-\log 2) .
\end{aligned}
$$

Although the central limit theorem yields in general a parabolic form $\psi(\Lambda) \propto\left(\Lambda-\Lambda^{\infty}\right)^{2}$ around the long-time average $\Lambda=\Lambda^{\infty}$, the Lyapunov exponent in this case, the above rate function does not contain any parabolic form due to the topological conjugacy to the Bernoulli shift without any fluctuation of the local expansion rate, which is always equal to $\log 2$. The magnetization-like function $\Lambda(q)$ has two non-analytic points at $q=1 / 2$ and $3 / 2$. Correspondingly, the rate function $\psi(\Lambda)$ has two linear parts with slopes of $d \psi / d \Lambda=1 / 2$ and $-1 / 2$. The former and latter correspond respectively to the $q$-phase transition caused by a diverging local expansion rate and by a crisis, in which the points $x=0 \pm \epsilon$ collide with the infinity points $x= \pm \infty$ on the asymptote $x_{t+1}=x_{t} / 2$. This simultaneous appearance of two $q$-phase transitions caused by a crisis and a diverging local expansion rate is also found in the logistic map $\left.y_{t+1}=4 y_{t}\left(1-y_{t}\right){ }^{?}\right)$

The simultaneous appearance of two q-phase transitions occurs frequently and is caused by crises such as a collision between a chaotic attractor and an unstable periodic orbit in non-hyperbolic dynamical systems, in which nonhyperbolicity originates from smooth extrema in the case of one-dimensional maps.?) Rigorous derivations of thermodynamic functions is in general impossible due to a singular invariant measure. One-dimensional maps that are topologically conjugate to the symmetric tent map or the Bernoulli shift listed in the literature?) are often called "fully-developed chaos" and these invariant densities are smooth functions, so that the thermodynamic functions can be analytically derived as shown in this short paper.

This work was supported by JSPS KAKENHI Grant Number 20540376. The author thanks Hiroki Hata and Takehiko Horita for illuminating discussions.

1) H. Mori and Y. Kuramoto: Dissipative Structures and Chaos (Springer, Berlin, 1998) Chap. 6 - Chap. 10D

2) E. N. Lorenz: J. Atmos. Sci. 20 (1963) 130.

3) P. Szépfalusy, T. Tél, and G. Vattay: Phys. Rev. A 43 (1991) 681.

4) R. L. Devaney: A First Course in Chaotic Dynamical Systems (Westview Press, Boulder, 1990) Chap. 13.

5) S. Katsura and W. Fukuda: Physica A 130 (1985) 597.

6) H. Mori, H. Hata, T. Horita, and T. Kobayashi: Prog. Theor. Phys. Suppl. 99 (1989) 1. 